\title{
Baseline characteristics associated with early visual acuity gains after ranibizumab treatment for retinal vein occlusion
}

\author{
W. Lloyd Clark ${ }^{1 *}$, Mimi Liu², John Kitchens ${ }^{3}$, Pin-wen Wang ${ }^{4}$ and Zdenka Haskova ${ }^{4}$
}

\begin{abstract}
Background: To identify baseline patient characteristics associated with early clinically significant visual acuity (VA) improvements within 3 months of treatment initiation in ranibizumab-treated patients with retinal vein occlusion (RVO) in the SHORE study.

Methods: Post hoc analysis of baseline patient characteristics in the randomized, open-label, vision examiner-masked SHORE phase 4 study that compared monthly versus pro re nata dosing of ranibizumab in patients with branch and central RVO. Patients who enrolled in SHORE fulfilled eligibility criteria per protocol $(N=202)$. SHORE data were retrospectively analyzed to identify baseline patient characteristics associated with early clinically significant improvements in VA, defined as improvement to a Snellen equivalent of 20/40 or better vision ( $\geq 69$ Early Treatment Diabetic Retinopathy Study [ETDRS] letters) or an increase in best-corrected VA (BCVA) of 15 or more ETDRS letters from baseline within 3 months of treatment initiation. Main outcome measures were BCVA gain of 15 or more ETDRS letters from baseline, Snellen equivalent of 20/40 or better vision, and baseline factors associated with early clinically significant improvement in BCVA.
\end{abstract}

Results: The median time for patients to achieve a BCVA of 20/40 or better was 59 days and the median time for patients to gain 15 or more ETDRS letters was 63 days. Better baseline BCVA (> 50 ETDRS letters/Snellen equivalent $\geq 20 / 100$ ), greater baseline total macular volume $\left(>9.99 \mathrm{~mm}^{3}\right)$, and presence of subretinal fluid at baseline were all associated with early improvement to 20/40 or better vision (ETDRS equivalent $\geq 69$ letters; $P<.0001, P=.02$, and $P=.03$, respectively).

Conclusions: This retrospective analysis found that better BCVA, greater total macular volume, and presence of subretinal fluid at baseline were associated with more rapid vision gains. Clinicians may find these helpful when considering the likelihood of achieving early clinically significant VA improvements with ranibizumab in patients with RVO.

Trial registration: ClinicalTrials.gov NCT01277302.

Keywords: Retinal vein occlusion, RVO, Branch retinal vein occlusion, BRVO, Central retinal vein occlusion, CRVO, Anti-VEGF, Ranibizumab

\section{Background}

Retinal vein occlusion (RVO) is a common cause of retinal vascular disease [1] and both branch RVO (BRVO) and central RVO (CRVO) are associated with vision loss and decreased vision-related quality of life [2, 3]. Although

\footnotetext{
* Correspondence: Iclark@palmettoretina.com

Presented at: Portions of these data were presented at the 47th Retina Society Annual Scientific Meeting, September 11-14, 2014, Philadelphia, PA, and the Association for Research in Vision and Ophthalmology 2015 Annual Meeting, May 3-7, 2015, Denver, CO.

'Palmetto Retina Center, 124 Sunset Court, West Columbia, SC 29169, USA Full list of author information is available at the end of the article
}

RVO is most prevalent in older individuals, it also can occur in younger individuals [4], potentially compromising their ability to work and drive. The introduction of antivascular endothelial growth factor (VEGF) agents to treat macular edema secondary to RVO was an important advancement for the improvement of visual outcomes in patients with BRVO and CRVO [5]. Despite these advances, understanding and identifying the patients who will benefit the most and experience rapid vision improvement from anti-VEGF treatment remains an open and important question.

(c) The Author(s). 2019 Open Access This article is distributed under the terms of the Creative Commons Attribution 4.0 International License (http://creativecommons.org/licenses/by/4.0/), which permits unrestricted use, distribution, and 
The randomized sham injection-controlled phase 3 BRAVO and CRUISE studies established the safety and efficacy of monthly treatment with the anti-VEGF agent ranibizumab for macular edema secondary to BRVO and CRVO, respectively [6-9]. The follow-up phase 4 SHORE study compared the efficacy of monthly and pro re nata (PRN) ranibizumab dosing on visual acuity (VA) maintenance in patients with BRVO and CRVO following disease activity stabilization [10]. The robust and clinically significant vision gains with ranibizumab during the initial 7-month-long monthly dosing period in SHORE were maintained over time with monthly and PRN dosing. Mean best-corrected VA (BCVA) gains from baseline at month 15 were 21.0 and 18.7 Early Treatment Diabetic Retinopathy Study (ETDRS) letters in the ranibizumab PRN and monthly arms, respectively [10].

The timing of patient responses to ranibizumab treatment in SHORE was heterogeneous. While the majority of patients met the prespecified disease stability criteria within 2 months of the end of the mandatory monthly loading phase (month 7 or month 8 of the study), a small number of patients failed to meet the disease stability criteria at any point during the study $(n=13)$ [10]. Although several baseline characteristics have been associated with an increased likelihood of VA improvements in patients with RVO treated with anti-VEGF agents in the limited literature available, the findings vary across studies and agents [11-13].

This retrospective exploratory analysis of SHORE data was designed to identify baseline predictors associated with early clinically significant VA improvement, defined as an increase in BCVA of 15 or more ETDRS letters from baseline or improvement to a Snellen equivalent of $20 / 40$ or better vision, the threshold required to hold an unrestricted driver's license across most of the United States. Understanding which baseline characteristics are associated with positive VA outcomes may help to inform both treatment expectations and approaches for patients with RVO.

\section{Methods}

This was a post hoc analysis of data from the SHORE study, the methods of which have been published previously [10]. Briefly, SHORE was a 15 -month, phase 4 , multicenter, randomized, open-label study of patients with BRVO or CRVO (ClinicalTrials.gov identifier: NCT01277302; $N=202$ ). The protocol was prospectively approved by the institutional review board at each study site, and all patients provided written informed consent. The study was conducted according to the International Conference on Harmonisation E6 Guideline for Good Clinical Practice and any national requirements. At enrollment, all study eyes had macular edema involving the foveal center due to BRVO or CRVO diagnosed within 12 months prior to screening. Key inclusion criteria for the study eye included ETDRS Protocol BCVA of 20/40-20/320 (Snellen equivalent). In SHORE, patients received 7 monthly ranibizumab $0.5 \mathrm{mg}$ injections at months $0-6$. Starting at the month 7 visit, each patient was assessed for potential randomization into the ranibizumab $0.5 \mathrm{mg}$ monthly and ranibizumab $0.5 \mathrm{mg}$ PRN treatment arms based on protocol-specified VA and anatomic disease stability criteria. Patients who did not meet the stability criteria were not randomized and continued to receive monthly ranibizumab $0.5 \mathrm{mg}$ injections until study completion. Disease stability criteria and PRN re-treatment criteria have been previously reported by Campochiaro et al. [10].

The main objective of this exploratory analysis of SHORE was to identify baseline characteristics predictive of early visual improvements. The analyses were designed to determine the predictors of early vision gains in a wide group of patients who experienced meaningful improvements during the initial treatment period. Rather than focusing on a single preset time point, we examined the prevalence of the endpoints over time to define the timing of vision improvements across the population. To more closely mirror clinical practice, where patients present for their next office visits at variable times, we first determined the median time to clinically significant vision gains and then followed with the predictor analyses. We performed predictor analyses of the following visual outcomes: (1) time from baseline to first achievement of Snellen equivalent of 20/40 or better vision, and (2) time to first gain of 15 or more ETDRS letters from baseline. The baseline demographic and disease-associated characteristics that were evaluated for potential predictive value are summarized in Table 1 . Baseline retinal nonperfusion status was not assessed for predictive value because more than $95 \%$ of patients had retinal nonperfusion at baseline, making a balanced sample size between present and absent groups impossible. Baseline predictor analyses of achievement of 20/40 or better vision excluded 22 (10.9\%) patients who had 20/ 40 or better vision at baseline.

Time to first Snellen equivalent of $20 / 40$ or better vision in the study eye (excluding eyes with $20 / 40$ or better vision at baseline) and first gain of 15 or more ETDRS letters from baseline were analyzed using the Kaplan-Meier method. The log-rank test was used to compare the distribution of time to endpoints between baseline predictors. Median time to events was provided by baseline predictor groups. Baseline predictors evaluated were age, sex, race, body mass index, baseline disease type, months since RVO diagnosis, smoking status, hypertension, diabetes, prior therapies for RVO, central subfield thickness, subretinal fluid, total macular volume, edema, cystoid space, retinal hemorrhage, diffuse edema, 


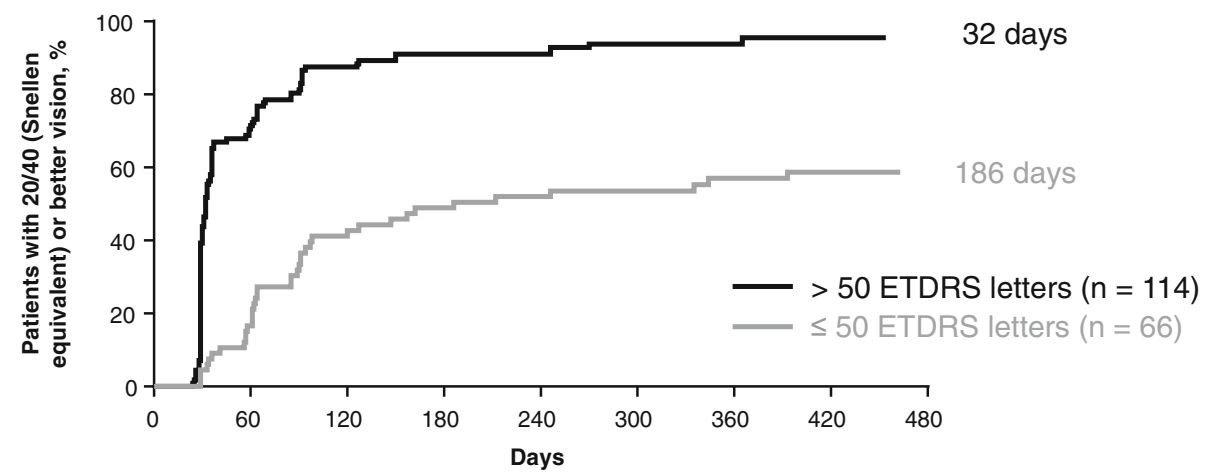

Fig. 1 Time to achievement of 20/40 or better vision by baseline best-corrected visual acuity. Excludes 22 (10.9\%) patients with 20/40 or better vision at baseline. ETDRS, Early Treatment Diabetic Retinopathy Study

potential interest emerged. First, male sex was associated with an earlier first gain of 15 or more ETDRS letters. Male patients gained 15 or more ETDRS letters in a median of 58 days compared with 92 days for female patients $(P=.03)$. The second characteristic of potential interest was worse BCVA at baseline. With a 43-day difference between the groups, patients with worse BCVA $(\leq 50$ ETDRS letters) at baseline exhibited a notably shorter median time to first gain of 15 or more ETDRS letters compared with patients with better BCVA (>50 ETDRS letters) at baseline (49 vs 92 days, respectively). This association, however, was not statistically significant $(P=.057)$.

\section{Discussion}

RVO is a serious disease that may lead to irreversible vision loss and permanent disability. Prompt treatment is critical to avoid irreparable loss of vision. Here, we discuss correlations between baseline patient characteristics and early VA improvements to help guide physicians' and patients' expectations at the start of treatment.

In SHORE, ranibizumab treatment of patients with BRVO and CRVO resulted in rapid improvements in
VA, with $39.0 \%$ of patients gaining 15 or more ETDRS letters from baseline after 1 injection, and $50.5 \%$ of patients achieving 20/40 or better vision after 1 injection. Rapid visual improvements are important both clinically and for patient quality of life. In clinical trials, the proportion of patients with vision gains of 15 or more ETDRS letters is a widely accepted clinically meaningful endpoint because gains of this magnitude represent a doubling of visual angle and correlate with the improvements in vision-related quality of life [14, 15]. Further, quick attainment of $20 / 40$ or better vision can have a significant impact on a patient's quality of life because it meets the VA threshold to obtain an unrestricted driver's license throughout most of the United States [16]. In this subanalysis, the median times to first gain of 15 or more ETDRS letters and first attainment of 20/40 or better vision were approximately 2 months, showing the rapid efficacy of ranibizumab treatment in a large proportion of patients with RVO.

When examined in the context of other ranibizumab RVO trials, the clinically significant vision gains in SHORE occurred 50 or more days more quickly than in

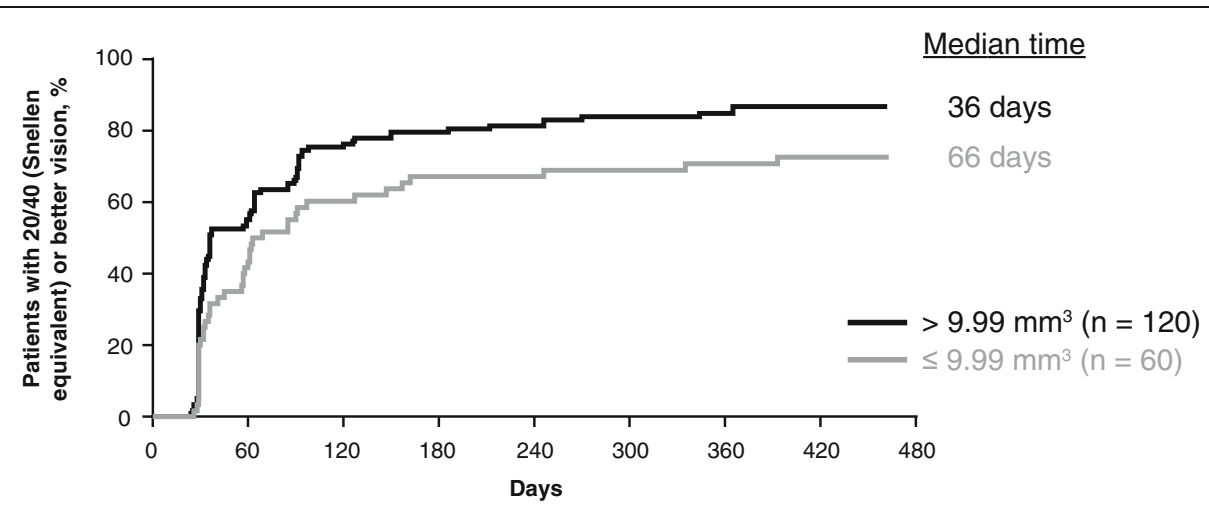

Fig. 2 Time to achievement of 20/40 or better vision by baseline total macular volume. Excludes 22 (10.9\%) patients with 20/40 or better vision at baseline; total macular volume was measured by the digital angiography reading center using spectral-domain optical coherence tomography 


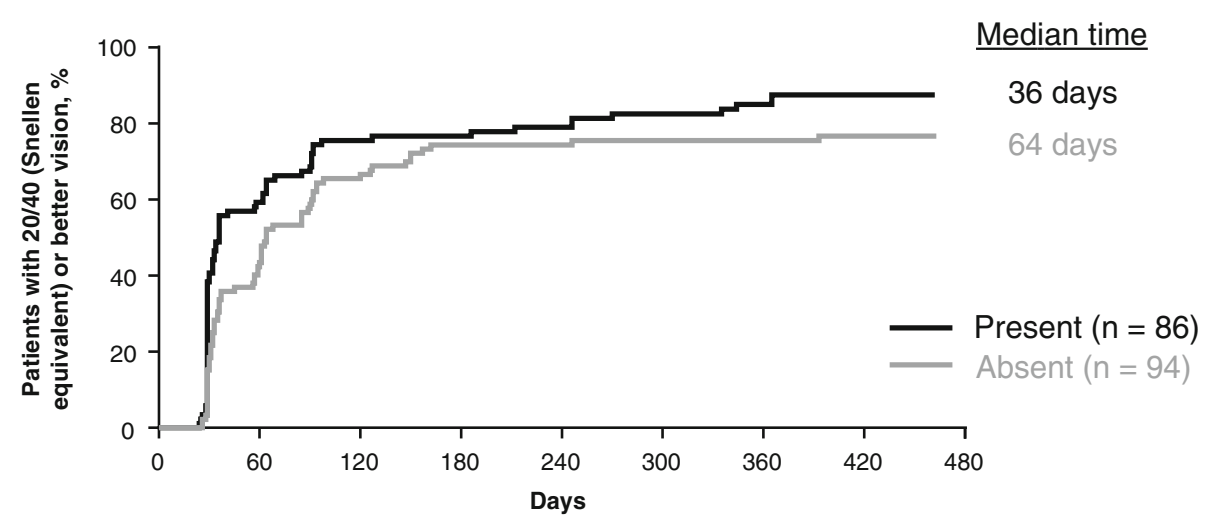

Fig. 3 Time to achievement of 20/40 or better vision by baseline subretinal fluid status. Excludes 22 (10.9\%) patients with 20/40 or better vision at baseline; subretinal fluid (presence or absence) by the digital angiography reading center was evaluated using spectral-domain optical coherence tomography

patients treated with ranibizumab $0.5 \mathrm{mg}$ in BRAVO and CRUISE [14]. Although the reason for the difference in time to clinically significant vision gains remains unclear, several differences in the study populations may have played a role in the varied findings, including differences in time from diagnosis, baseline vision, and baseline disease severity.

To better understand which patients with RVO are more likely to show rapid clinically significant vision gains with ranibizumab treatment, this SHORE subanalysis examined a wide variety of baseline characteristics for their ability to predict achievement of early visual improvements within 3 months of treatment initiation. Of the more than 20 demographic, systemic, visual, and ocular anatomic baseline characteristics examined, the 3 factors predictive of earlier $20 / 40$ or better vision were better BCVA ( $>50$ ETDRS letters; $P<.0001$ ), greater macular volume $(P=.02)$, and presence of subretinal fluid at baseline $(P=.03)$. Additional factors that were of potential interest for their association with earlier 15 or more ETDRS letter gains from baseline were male sex $(P=.03)$ and worse baseline BCVA ( $\leq 50$ ETDRS letters; $P=.057$ ).

The associations found between baseline BCVA status and early vision improvement were not surprising because baseline BCVA status played an important role in both VA metrics used in this analysis. For the early attainment of 20/40 or better vision metric, patients with better baseline BCVA (>50 ETDRS letters) had an advantage because they were closer to the 20/40 threshold, allowing them to achieve $20 / 40$ or better vision more quickly. In contrast, worse baseline vision ( $\leq 50$ ETDRS letters) provided an advantage for the early attainment of 15 or more ETDRS letters from baseline because patients with worse baseline vision had greater room for improvement compared with patients with better baseline vision.

The predictive value of greater baseline macular volume and presence of subretinal fluid at baseline for early vision improvements is also anatomically plausible given the mechanism of action of ranibizumab and the pathophysiology of macular edema secondary to RVO. Patients with greater macular volume at baseline likely had increased disease severity caused by vascular permeability and fluid accumulation in the macula, factors known to disrupt vision. Thus, ranibizumab treatment allowed for rapid edema reduction and subsequent vision gains. Similarly, the rapid effects of ranibizumab on vascular permeability likely contributed to the early vision gains observed in ranibizumab-treated patients with subretinal fluid at baseline. Although anatomic features associated with fluid retention were associated with early vision gains, others such as retinal hemorrhage were not. These data indicate that vision loss due to edema was more rapidly and easily recoverable with ranibizumab treatment than vision loss due to retinal damage.

In this subanalysis of the SHORE study, male sex was associated with an earlier gain of 15 or more ETDRS letters. This finding is likely explained by the demographics of the cohort because the proportion of male patients with a Snellen equivalent of 20/200 or worse vision at baseline $(22 / 118[18.6 \%])$ was nearly double that of female patients (8/84 [9.5\%]; Table 2; $P=.07)$. Further, male patients were slightly younger than female patients, and a higher proportion of male patients than female patients had the more visually debilitating diagnosis of CRVO. These differences could have created greater room for improvement in male patients, possibly resulting in faster VA improvement compared with female patients. Thus, it is unknown and perhaps unlikely that the finding that male sex was predictive of early visual improvements in patients with RVO treated with ranibizumab is generalizable to the overall population.

Reports of specific baseline characteristics associated with VA improvements in anti-VEGF-treated patients with RVO vary throughout the literature, and include 
Table 2 Baseline Characteristics in Male Versus Female Patients

\begin{tabular}{|c|c|c|}
\hline Characteristic & Male $(n=118)$ & Female $(n=84)$ \\
\hline Mean age, years (SD) & $64.0(12.7)$ & $69.5(11.3)$ \\
\hline \multicolumn{3}{|l|}{ Baseline occlusion type, n (\%) } \\
\hline BRVO/HRVO & $59(50.0)$ & $56(66.7)$ \\
\hline CRVO & $59(50.0)$ & $28(33.3)$ \\
\hline Mean BCVA, ETDRS letters (SD) & $52.2(14.0)$ & $55.0(11.6)$ \\
\hline Patients with 20/200 or worse vision, n (\%) & $22(18.6)$ & $8(9.5)$ \\
\hline Mean CST, $\mu \mathrm{m}(\mathrm{SD})$ & $539.0(177.5)$ & $508.6(138.3)$ \\
\hline Mean time since diagnosis of RVO, months (SD) & $1.7(2.1)$ & $1.7(1.7)$ \\
\hline \multicolumn{3}{|l|}{ Prior therapies, n (\%) } \\
\hline Intravitreal steroids & $7(5.9)$ & $2(2.4)$ \\
\hline Laser photocoagulation & $1(0.8)$ & $1(1.2)$ \\
\hline \multicolumn{3}{|l|}{ Comorbidities, n (\%) } \\
\hline Open-angle glaucoma & $10(8.5)$ & $7(8.3)$ \\
\hline Hypertension & $70(59.3)$ & $61(72.6)$ \\
\hline Angina & $4(3.4)$ & $3(3.6)$ \\
\hline Congestive heart failure & $2(1.7)$ & $4(4.8)$ \\
\hline Diabetes mellitus & $18(15.3)$ & $13(15.5)$ \\
\hline
\end{tabular}

BCVA best-corrected visual acuity, BRVO branch retinal vein occlusion, CRVO central retinal vein occlusion, CST central subfield thickness, ETDRS Early Treatment Diabetic Retinopathy Study, HRVO hemiretinal vein occlusion, RVO retinal vein occlusion

baseline BCVA, younger age, and time to treatment $[11,12]$. Direct comparisons between analyses remain difficult due to the different parameters examined in each study. In particular, it is important to note that previous studies examined the effect of baseline characteristics on final VA, not the time to reach a clinically significant change in VA as examined in this SHORE subanalysis. In addition, clinical practice survey data indicate that the majority of patients with macular edema secondary to RVO are treated using a treat-and-extend or as-needed approach [17] instead of monthly, potentially limiting the generalizability of clinical trial findings.

This post hoc SHORE analysis of baseline predictors should be interpreted with caution because statistical analyses were not prespecified and this analysis may not have been sufficiently powered for all baseline predictor endpoints. In addition, because this was a post hoc analysis, baseline groups analyzed were not evenly distributed. For example, some of the predictor analyses had a distribution up to 2:1 between the compared groups (ie, total macular volume and baseline BCVA).

Despite these limitations, this SHORE analysis provides important hypotheses on the characteristics that may predict early treatment response in patients with macular edema secondary to BRVO and CRVO. The key baseline predictors of early VA improvement within 3 months of treatment initiation found in this analysis were baseline BCVA of more than 50 ETDRS letters, baseline macular volume greater than $9.99 \mathrm{~mm}^{3}$, and presence of subretinal fluid. A prospective study would be needed to confirm these hypotheses. Recognizing the baseline factors associated with early VA response to ranibizumab treatment in patients with BRVO and CRVO may help guide patients' and physicians' expectations at the start of therapy.

\section{Abbreviations}

BCVA: Best-corrected visual acuity; BMI: Body mass index; BRVO: Branch retinal vein occlusion; CRVO: Central retinal vein occlusion; CST: Central subfield thickness; DBP: Diastolic blood pressure; ETDRS: Early Treatment Diabetic Retinopathy Study; HRVO: Hemiretinal vein occlusion; PRN: Pro re nata; RVO: Retinal vein occlusion; SBP: Systolic blood pressure; VA: Visual acuity; VEGF: Vascular endothelial growth factor

\section{Acknowledgements}

Funding was provided by Genentech, Inc., a member of the Roche Group, for third-party writing assistance, which was provided by Betsy C. Taylor, PhD, CMPP, of Envision Pharma Group.

\section{Funding}

Funding for this study was provided by Genentech, Inc., South San Francisco, CA. Genentech, Inc., South San Francisco, CA, participated in the design and conduct of the studies; data collection, analysis, and interpretation of results; and preparation, review, and approval of the manuscript.

\section{Availability of data and materials}

Qualified researchers may request access to individual patient-level data through the clinical study data request platform (www.clinicalstudydatarequest.com). Further details on Roche's criteria for eligible studies are available here (https://clinicalstudydatarequest.com/Study-Sponsors/Study-SponsorsRoche.aspx). For further details on Roche's Global Policy on the Sharing of Clinical Information and how to request access to related clinical study documents, see here (https://www.roche.com/research_and_development/who_we_are_ how_we_work/clinical_trials/our_commitment_to_data_sharing.htm). 


\section{Authors' contributions}

Research design: WLC, ML, JK, P-wW, and ZH. Data acquisition and/or research execution: P-wW and ZH. Data analysis and/or interpretation: WLC, ML, JK, PwW, and ZH. Manuscript preparation: WLC, ML, JK, P-wW, and ZH. All authors read and approved the final version of this manuscript.

\section{Ethics approval and consent to participate}

This study includes research conducted on human subjects. The study protocol was prospectively approved by the institutional review board at each study site, and the study was conducted according to the International Conference on Harmonisation E6 Guideline for Good Clinical Practice and any national requirements. Patients provided written, informed consent for the trial and for future medical research and analyses based on results of the trial. The existing data from this clinical trial were assessed retrospectively by exploratory analyses. The study sponsor, Genentech, Inc., granted permission to use the data. No patient identifiable information was connected with the datasets used for these retrospective analyses.

\section{Consent for publication}

Not applicable.

\section{Competing interests}

The authors have made the following disclosures: WLC has received grant support and served on the speakers' bureau and as a consultant for Genentech, Inc./Roche (South San Francisco, CA) and Regeneron Pharmaceuticals (Tarrytown, NY); has received travel support and honoraria from Bayer (Leverkusen, Germany); has received grant support and served as a consultant for Santen (Osaka, Japan); and has received grant support from Allergan (Dublin, Ireland). ML has no financial conflicts of interest to disclose. JK has served as a consultant for Alcon (Forth Worth, TX), Allergan (Dublin, Ireland), Genentech, Inc. (South San Francisco, CA), Regeneron (Tarrytown, NY), and ThromboGenics (Leuven, Belgium); and has served as a consultant for and holds stock in Synergetics (O'Fallon, MO). P-wW and ZH are employees of Genentech, Inc. (South San Francisco, CA).

\section{Publisher's Note}

Springer Nature remains neutral with regard to jurisdictional claims in published maps and institutional affiliations.

\section{Author details}

'Palmetto Retina Center, 124 Sunset Court, West Columbia, SC 29169, USA.

${ }^{2}$ Colorado Retina Associates, Denver, CO, USA. ${ }^{3}$ Retina Associates of Kentucky, Lexington, KY, USA. ${ }^{4}$ Genentech, Inc., South San Francisco, CA, USA.

Received: 25 July 2018 Accepted: 14 December 2018

Published online: 08 January 2019

\section{References}

1. Rogers S, McIntosh RL, Cheung N, Lim L, Wang JJ, Mitchell P, et al;; International Eye Disease Consortium. The prevalence of retinal vein occlusion: pooled data from population studies from the United States, Europe, Asia, and Australia. Ophthalmology. 2010;117:313-9 e1.

2. Awdeh RM, Elsing SH, Deramo VA, Stinnett S, Lee PP, Fekrat S. Vision-related quality of life in persons with unilateral branch retinal vein occlusion using the 25-item National Eye Institute Visual Function Questionnaire. Br J Ophthalmol. 2010;94:319-23.

3. Deramo VA, Cox TA, Syed AB, Lee PP, Fekrat S. Vision-related quality of life in people with central retinal vein occlusion using the 25-item National Eye Institute Visual Function Questionnaire. Arch Ophthalmol. 2003;121:1297-302.

4. Laouri M, Chen E, Looman M, Gallagher M. The burden of disease of retinal vein occlusion: review of the literature. Eye (Lond). 2011;25:981-8.

5. Ho M, Liu DT, Lam DS, Jonas JB. Retinal vein occlusions, from basics to the latest treatment. Retina. 2016;36:432-48.

6. Brown DM, Campochiaro PA, Bhisitkul RB, Ho AC, Gray S, Saroj N, et al. Sustained benefits from ranibizumab for macular edema following branch retinal vein occlusion: 12-month outcomes of a phase III study. Ophthalmology. 2011;118:1594-602.

7. Brown DM, Campochiaro PA, Singh RP, Li Z, Gray S, Saroj N, et al.; CRUISE Investigators. Ranibizumab for macular edema following central retinal vein occlusion: six-month primary end point results of a phase III study. Ophthalmology. 2010;117:1124-33e1.

8. Campochiaro PA, Brown DM, Awh CC, Lee SY, Gray S, Saroj N, et al. Sustained benefits from ranibizumab for macular edema following central retinal vein occlusion: twelve-month outcomes of a phase III study. Ophthalmology. 2011;118:2041-9.

9. Campochiaro PA, Heier JS, Feiner L, Gray S, Saroj N, Rundle AC, et al.; BRAVO Investigators. Ranibizumab for macular edema following branch retinal vein occlusion: six-month primary end point results of a phase III study. Ophthalmology. 2010;117:1102-12e1.

10. Campochiaro PA, Wykoff CC, Singer M, Johnson R, Marcus D, Yau L, et al. Monthly versus as-needed ranibizumab injections in patients with retinal vein occlusion: the SHORE study. Ophthalmology. 2014;121:2432-42.

11. Daien V, Navarre S, Fesler P, Vergely L, Villain M, Schneider C. Visual acuity outcome and predictive factors after bevacizumab for central retinal vein occlusion. Eur J Ophthalmol. 2012;22:1013-8.

12. Scott IU, VanVeldhuisen PC, Oden NL, Ip MS, Blodi BA, Hartnett ME, et al.; Standard Care versus COrticosteroid for REtinal Vein Occlusion Study Investigator Group. Baseline predictors of visual acuity and retinal thickness outcomes in patients with retinal vein occlusion: Standard Care versus COrticosteroid for REtinal Vein Occlusion Study report 10. Ophthalmology. 2011:118:345-52.

13. Jaissle GB, Szurman P, Feltgen N, Spitzer B, Pielen A, Rehak M, et al.; Retinal Vein Occlusion Study Group. Predictive factors for functional improvement after intravitreal bevacizumab therapy for macular edema due to branch retinal vein occlusion. Graefes Arch Clin Exp Ophthalmol. 2011;249:183-92.

14. Thach AB, Yau L, Hoang C, Tuomi L. Time to clinically significant visual acuity gains after ranibizumab treatment for retinal vein occlusion: BRAVO and CRUISE trials. Ophthalmology. 2014;121:1059-66.

15. Suñer IJ, Kokame GT, Yu E, Ward J, Dolan C, Bressler NM. Responsiveness of NEl VFQ-25 to changes in visual acuity in neovascular AMD: validation studies from two phase 3 clinical trials. Invest Ophthalmol Vis Sci. 2009;50:3629-35.

16. Steinkuller PG. Legal vision requirements for drivers in the United States. Virtual Mentor. 2010;12:938-40.

17. Rezaei KA. Global Trends in Retina. https://www.asrs.org/content/documents/ 2015_global_trends_in_retina_survey_-_for_website.pdf. Accessed 16 May 2018.

\section{Ready to submit your research? Choose BMC and benefit from:}

- fast, convenient online submission

- thorough peer review by experienced researchers in your field

- rapid publication on acceptance

- support for research data, including large and complex data types

- gold Open Access which fosters wider collaboration and increased citations

- maximum visibility for your research: over $100 \mathrm{M}$ website views per year

At BMC, research is always in progress.

Learn more biomedcentral.com/submissions 